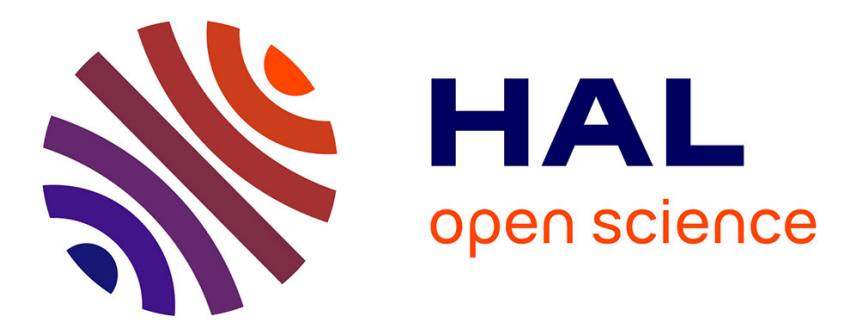

\title{
Sur une méthode altimétrique d'analyse morphologique appliquée à la Bretagne péninsulaire
}

\author{
H. Baulig
}

\section{To cite this version:}

H. Baulig. Sur une méthode altimétrique d'analyse morphologique appliquée à la Bretagne péninsulaire. Bulletin de l'Association de géographes français, 1926, 10, pp.7-9. 10.3406/bagf.1926.8307 . insu-01778249

\section{HAL Id: insu-01778249 \\ https://hal-insu.archives-ouvertes.fr/insu-01778249}

Submitted on 4 Mar 2021

HAL is a multi-disciplinary open access archive for the deposit and dissemination of scientific research documents, whether they are published or not. The documents may come from teaching and research institutions in France or abroad, or from public or private research centers.
L'archive ouverte pluridisciplinaire HAL, est destinée au dépôt et à la diffusion de documents scientifiques de niveau recherche, publiés ou non, émanant des établissements d'enseignement et de recherche français ou étrangers, des laboratoires publics ou privés.

\section{()ㅜ) $\Theta$}

Distributed under a Creative Commons Attribution - NoDerivatives| 4.0 International 


\section{Sur une méthode altimétrique d'analyse morphologique appliquée à} la Bretagne péninsulaire

Henri Baulig

\section{Citer ce document / Cite this document :}

Baulig Henri. Sur une méthode altimétrique d'analyse morphologique appliquée à la Bretagne péninsulaire. In: Bulletin de l'Association de géographes français, №10, 3e année,1926. pp. 7-9;

doi : https://doi.org/10.3406/bagf.1926.8307

https://www.persee.fr/doc/bagf_0004-5322_1926_num_3_10_8307

Fichier pdf généré le 25/04/2018 
bien mort, ou que seule l'agriculture en conserve de faibles vestiges en quelques coins arriérés (Bretagne intérieure), le Bocage normand a retenu quelque chose de sa vie d'antan; mais, chose exceptionnelle, c'est non l'agriculture, mais l'industrie qu'affectent ces survivances.

\section{Discussion}

M. Allix est frappé des ressemblances du genre de vie décrit. avec celui de certains massifs alpins (industries locales, colpor(age, etc.).

\section{Communication de H. Haulir.}

\section{Sur une méthode altimétrique d'analyse morphologique appliquée da la Bretagne péninsulaire}

On s'est proposé de rechercher une méthode, soil graphique, soit statistique, propre à révéler, d'une manière aussi objective IIue possible, les discontinuités topographiques ef en particulier les surfaces d'aplanissement étendues.

La courbe hypsographique de Penck ne satisfait pas a cette ondition, car elle représente l'étendue des zones hypsométriques et non les pentes de la surface, et d'ailleurs les points d'inflexion sont difficileś à préciser. Il y a intérêt à la remplacer par une courhe analogue is une courle de frequence, constluite en portant les longueurs proportionnelles aux aires des zontes hypsometriques non pas bout à bout, mais à partir d'une même origine. Les masima correspondant aux régions d'aplanissement se traduisent alors par des saillants, et les minima, par des rentrants de la rourbe.

Néanmoins, la méthode suppose l'existence d'une carte en courbes de niveau. La mesure des aires reste une opération délicate; et enfin la précision des résultats ne peul dépasser sensiblement l'équidistance. On peut donc se demander s'il ne serait pas possible, partant d'une carte en hachures, de remplacer la mesure des aires par le dénombrement des cotes. Coladoit être possible, a condition :

1 - Que les coles soient exactes; or celles de la carte au $80.000^{e}$ pour la région considérée sont affectées d'une erreur probable de quelques mètres; mais cette erreur étant, de toute vraisemblance, accidentelle, s’atténue dans les moyennes, et d'autant plus que celles-ci reposent sur un plus grand nombre de cctes; 2० Que les cotes soient uniformément réparties, sans égard aux 
particularités de la topographic. Or, on sait que les topographes cotent de préférence les points hauts, puis les thalwegs, et que les yersants sont, sacrifiés; par conséquent, les surfaces aplanies seront exagérées, mais. cela n'a pas d'inconvénient quand on se propose de déterminer non l'étendue des différentes zones hypsométriques, mais les altitudes vers lesquelles se produisent les principaux aplanissements;

30 Que les cotes soient suffisamment denses, eu égard à la fo is a l'étendue de la région et à son relief : l'expérience seule permet d'en décider. La région considérée porte 11.800 cotes sur 20.700 kilomètres carrés, soit 57 cotes par 100 kilomètres carrés.

Afin de faire disparaitre, en partic au moins, les menues irrégularités de la courbe, on recourt à un procédé de nivellement graduel dont voici le principe $: a, b ; c, d$, e étant les nombres $q u^{i}$ représentent la fréquence de cinq cotes consécutives, on remplace $c$ par la moyenne arithmétique de ces cinq nombres: de même, on remplace a par lis inoyenne arithmótique de $b, d, d, e$, $f$ ete. Cela fait, on se lrouve en présence dune courbe plus régulière, présentant de menues identations qu'on peut croire fortuites et des inflexions plus larges qu'on peut présumer systématiques. Mais comment distinguer?

Il ne parait pas possible de lrater la question mathématiquement, faute de pouvoir la poser en termes rigoureux. Mais on peut admettre qu'un maximum net, encadré entre deux minima également nets, apparaissant ì la même altitude sur des rourbes partielles correspondent à des portions entièrement différentes de la région, traduit, très vraisemblablement, une réalité, et la probabilité confine à la certitude quand cette coüncidence se répète à différentes altitudes. Cela suppose, bien entendu, que les différentes parties de la région ont évolué solidairement et sans aucune déformation, ce dont la méthode employée permet précisément de décider.

La Bretagne péninsulaire a donc été divisée en une zone $\mathrm{N}$ et une zone $S$ par le parallèle de 53; 50 et en trois zones $E$, Centre et $W$, définies par les méridiens de 5r, de $6 r$ et de $7^{i}$, et $l^{\prime}$ 'on a construit les courbes partielles pour chacune de ces zones.

A ne considérer que les inflexions bien nettes et apparaissant à la fois sur les courbes $N$ et $S$ et en outre sur deux au moins des trois autres courbes, on arrive à reconnaître les maxima suivants : $128 \mathrm{~m}$; $90 \mathrm{~m}$. (maximum principal, figurant sur toutes les courbes, décomposé peut-être par un minimum relatif à $90 \mathrm{~m}$. en deux maxima à 94 et $86 \mathrm{~m}$.); $56 \mathrm{~m}$. (très net, figure sur toutes les courbes, à quelques mètres près); $45 \mathrm{~m}$. (ou plutôt, très probablement, double maximum à 51 et $42 \mathrm{~m}$. avec minimum relatif à $45 \mathrm{~m}$.); 
$25 \mathrm{~m}$. (peu marqué); $10 \mathrm{~m}$. (douteux). Il existe, très probablement, d'autres coïncidences significatives au-dessus de $128 \mathrm{~m}$. : mais elles sont masquées par les variations accidentelles.

Traduits en langage morphologique, ces résultats signifient : $1^{\circ}$ qu'il existe, dans les différentes parties de la Bretagne, de larges surfaces aplanies au voisinage immédiat des maxima indiqués et un peu au-dessus; $2^{\circ}$ que ces surfaces n'étant évidemment ni structurales ni construites, sont des surfaces d'érosion cyclique; $3 \circ$ que, depuis le cycle de $128 \mathrm{~m}$. au moins, le passage d'un cycle au suivant s'est fait sans dénivellation appréciable des diverses parties, le degré d'approximation de cette conclusion étant de l'ordre du mètre ou de quelques mètres au plus. De là paraissent devoir découler des conclusions soit eustatiques, soit isostatiques rigoureuses.

Mais on ne saurait, s'appuyant sur de simples concordances approximatives d'altitude, paralléliser d'emblée ces niveaux d'érosion avec les terrasses alluviales de $M$. Depéret, car le nivellement d'une surface en roche dure et la construction d'une plaine alluviale sont deux phénomènes profondément dissemblables et qui n'ont pour ainsi dire aucune commune mesure dans le temps.

Ces conclusions ne préjugent pas davantage la question de l'existence, en Bretagne, d'une "pénéplaine " générale, antérieure aux niveaux dont il est question ici.

La présente méthode ne parait applicable qu'à des régions assez vastes, de relief modeste, exemptes de surfaces structurales ou construites étendues, et enfin non atteintes par les déformations isostatiques liées aux glaciations quatermaires.

\section{Discussion}

M. Mussef élève des doutes sur la valeur des conclusions tirées de la statistique altimétrique. Il déclare ne pas voir sur le terrain les surfaces ainsi définies.

Visite de l'Agence généhale oes Colonies 22 auril 1926

M. Guilaume Capus, Docteur ès sciences, Conseiller technique de l'Agence générale des Colonies, accueille les membres de l'A. G. F. dans les galeries du Palais-Royal et, grâce à ses explications, cette visite de produits coloniaux prend tout son intérêt scien: 